\title{
Exact Solution of the Schrödinger Equation with a Central Potential
}

\author{
E. J. Kanellopoulos, Th. V. Kanellopoulos, and K. Wildermuth
}

Institut für Theoretische Physik der Universität Tübingen

Received March 31/October 15, 1971

\begin{abstract}
The exact solution of the Schrödinger equation is derived for the case of a central potential under rather weak restriction on it. The solution is given in a form of a simple series which converges strongly and it is suitable for calculation of phase shifts and eigenvalues. Also, as the derivation of the solution is purely algebraic its analytical continuation in the energy or angular momentum complex plane is straightforward.
\end{abstract}

\section{Introduction}

In Ref. [1] an "integro-iteration" method was used to obtain the solution of any second order linear differential equation. This method when applied to the Schrödinger equation gives the solution under rather weak restrictions on the potential $V(r)$.

The procedure starts with the usual transformation of the differential equation into an integral one. Then the function is split in two components, thus obtaining two coupled integral equations. At this point an integration step is inserted and then a two component iteration is performed. The solution is obtained in terms of a simple series which is strongly convergent for a large class of potentials.

Although the modification of the procedure for solving the integral equation by a simple iteration looks to be slight, its effect on the result is quite remarkable. To be more explicit: the simple iteration method leads to the Born's expansion. However, as, for $l>0$, the kernel has two components, the iteration gives multiple series, i.e. the $n^{\text {th }}$ term of it has $2^{n}$ components. Besides one has to face difficulties with its convergence. On the other hand the Fredholm method is too powerful to be used in the case of a simple second order linear differential equation and bears comparatively too much complexity. For example one has to compute two series in each of which the $n^{\text {th }}$ term has $n$ ! components. Moreover it seems to demand stronger limitations on the potential than the present method, i.e. as one can see in [2] the minimum conditions on $V(r)$ using 
Fredholm's method are

$$
\int_{0}^{\infty} d r \cdot r|V(r)|<\infty
$$

and

$$
\int_{0}^{\infty} d r \cdot r^{2}|V(r)|<\infty
$$

while in the present method even condition (a) is more than enough.

In Part I we give briefly the results we need here from Ref. [1]. In Part II the solution for the bound state problem is given and the eigenvalue equation derived. It is found that for both types of solutions the sufficient conditions on the potential for their convergence are

$$
\begin{aligned}
& \int_{0}^{a} r|V(r)| d r<\infty \\
& \int_{a}^{\infty}|V(r)| d r<\infty
\end{aligned}
$$

for any $0<a<\infty$. The same conditions we have in case of scattering problems for the Type I solution. The second condition just leaves out the Coulomb potential. In Part III we treat the case of continuum spectrum. In this case the problem is an initial value one and the only condition which is imposed on the solution is that the radial part of the wave function is regular at the origin. This is enough to define the solution apart from an arbitrary multiplier which is irrelevant. The asymptotic behaviour of the radial part is derived. Therefore one obtains the expressions for phase shifts and the scattering amplitude directly without introducing the Jost functions [3].

\section{Formulation}

Let

$$
z^{\prime \prime}+B(x) z=C(x) z
$$

be the equation to be solved.

If $u_{1}$ and $u_{2}$ are two independent solutions of

$$
u^{\prime \prime}+B(x) u=0
$$

and

$$
W\left(u_{1}, u_{2}\right)=u_{1} u_{2}^{\prime}-u_{1}^{\prime} u_{2}=1
$$


then one solution of (1) is given by

(i) Type I

$$
\begin{gathered}
z=u_{1} e^{-f(x)} \Phi_{1}\left(\begin{array}{c}
x \\
a, a
\end{array}\right)+u_{2} e^{f(x)} \int_{a}^{x} C u_{1}^{2} e^{-2 f\left(x^{\prime}\right)} \Phi_{1}\left(\begin{array}{c}
x^{\prime} \\
a, a
\end{array}\right) d x^{\prime} \\
\text { where } \\
f(x)=\int^{x} C u_{1} u_{2} d x^{\prime}
\end{gathered}
$$

and $\Phi_{1}\left(\begin{array}{c}x \\ \mathrm{a}, \mathrm{a}\end{array}\right)$ a simple series generated by:

$$
\Phi_{1}\left(\begin{array}{c}
x \\
a, a
\end{array}\right)=1-\int_{a}^{x} C u_{2}^{2} e^{2 f} d x^{\prime} \int_{a}^{x^{\prime}} C u_{1}^{2} e^{-2 f} \Phi_{1}\left(\begin{array}{c}
x^{\prime \prime} \\
a, a
\end{array}\right) d x^{\prime \prime}
$$

or

(ii) Type II

provided that

$$
z=-u_{2} \int_{a}^{x} \frac{F_{1}\left(\begin{array}{c}
x^{\prime} \\
a, a
\end{array}\right)}{u_{2}^{2}} d x^{\prime}
$$

$$
u_{1}(a)=0 \text {. }
$$

Here

$$
F_{1}\left(\begin{array}{c}
x \\
a, a
\end{array}\right)=1+\int_{a}^{x} C u_{2}^{2} d x^{\prime} \int_{a}^{x^{\prime}} \frac{F_{1}\left(\begin{array}{c}
x^{\prime \prime} \\
a, a
\end{array}\right)}{u_{2}^{2}} d x^{\prime \prime}
$$

$\Phi_{1}\left(\begin{array}{c}x \\ a, a\end{array}\right)$ and $F_{1}\left(\begin{array}{c}x \\ a, a\end{array}\right)$ are connected through the relation

$$
\Phi_{1}\left(\begin{array}{c}
x \\
a, a
\end{array}\right)=e^{{ }_{\int}^{x} C u_{1} u_{2} d \xi} F_{1}\left(\begin{array}{c}
x \\
a, a
\end{array}\right) .
$$

For the convergence of the series given by (6) and (9) we shall be satisfied with the sufficient conditions:

$$
\begin{aligned}
& \bar{q}=\int_{a}^{x}\left|C u_{2}^{2} e^{2 f}\right| d x^{\prime} \int_{a}^{x^{\prime}}\left|C u_{1}^{2} e^{-2 f}\right| d x^{\prime \prime}<M<\infty, \\
& \bar{p}=\int_{a}^{x}\left|C u_{2}^{2}\right| d x^{\prime} \int_{a}^{x^{\prime}}\left|\frac{1}{u_{2}^{2}}\right| d x^{\prime \prime}<N<\infty
\end{aligned}
$$

for any $x$ in the region of interest.

\section{Initial Value Problems}

If we seek for a solution for which

$$
z(a)=0
$$

17 Commun. math. Phys., Vol. 24 
it is enough to choose $u_{1}$ such that

$$
u_{1}(a)=0
$$

With this one condition the solution is defined apart from a constant factor which is irrelevant.

\section{Boundary Value Problems}

If we want a solution $z$ with the conditions

$$
\begin{aligned}
& z(a)=0 \\
& z(b)=0
\end{aligned}
$$

and

and we find as eigenvalue equation either

$$
u_{1}(a)=u_{2}(b)=0
$$

or

$$
\begin{aligned}
& \Phi_{1}\left(\begin{array}{c}
b \\
a, a
\end{array}\right)=0 \\
& F_{1}\left(\begin{array}{c}
b \\
a, a
\end{array}\right)=0 .
\end{aligned}
$$

Note that, because of (10), (15) and (15a) are equivalent.

\section{Bound State Problems}

1. The radial part of the Schrödinger equation, after putting $w_{l}(r)$ $=r R_{l}(r)$ becomes

$$
\left[\frac{d^{2}}{d r^{2}}-\kappa^{2}-\frac{l(l+1)}{r^{2}}\right] w_{l}(r)=-U(r) w_{l}(r)
$$

where

$$
\kappa^{2}=\frac{2 m|E|}{\hbar^{2}}, \quad U(r)=\frac{2 m}{\hbar^{2}} V(r) .
$$

We choose two independent solutions of the unperturbed equation

$$
\begin{aligned}
& u_{1}(r)=S_{l}(r)=\sqrt{\frac{\pi r}{2}} I_{l+\frac{1}{2}}(\kappa r) \\
& u_{2}(r)=C_{l}(r)=-\sqrt{\frac{2 r}{\pi}} K_{l+\frac{1}{2}}(\kappa r)
\end{aligned}
$$

where $I_{l+\frac{1}{2}}$ and $K_{l+\frac{1}{2}}$ are modified Bessel functions defined as in [4]. 
Then we have

$$
u_{1}(0)=S_{l}(0)=0, \quad u_{2}(\infty)=C_{l}(\infty)=0
$$

and

$$
W\left(S_{l}, C_{l}\right)=1 \text {. }
$$

The wave function is given either by

(a) $\quad w_{l}(r)=S_{l} e^{f(r)} \Phi_{1}\left(\begin{array}{c}r \\ 0,0\end{array}\right)-C_{l} e^{-f(r)} \int_{0}^{r} U\left(r^{\prime}\right) S_{l}^{2}\left(r^{\prime}\right) e^{2 f\left(r^{\prime}\right)} \Phi_{1}\left(\begin{array}{c}r^{\prime} \\ 0,0\end{array}\right) d r^{\prime}$

or by

(b) $\quad w_{l}(r)=-C_{l}(r) \int_{0}^{r} \frac{F_{1}\left(\begin{array}{c}r^{\prime} \\ 0,0\end{array}\right)}{C_{l}^{2}\left(r^{\prime}\right)} d r^{\prime}$

where

$$
\begin{aligned}
& \Phi_{1}\left(\begin{array}{c}
r \\
0,0
\end{array}\right)=1-\int_{0}^{r} U C_{l}^{2} e^{-2 f} d r^{\prime} \int_{0}^{r^{\prime}} U S_{l}^{2} e^{2 f} \Phi_{1}\left(\begin{array}{c}
r^{\prime \prime} \\
0,0
\end{array}\right) d r^{\prime \prime} \\
& F_{1}\left(\begin{array}{c}
r \\
0,0
\end{array}\right)=1-\int_{0}^{r} U C_{l}^{2} d r^{\prime} \int_{0}^{r^{\prime}} \frac{F_{1}\left(\begin{array}{c}
r^{\prime \prime} \\
0,0
\end{array}\right)}{C_{l}^{2}} d r^{\prime \prime}
\end{aligned}
$$

and $\quad f(r)=\int_{0}^{r} U S_{l} C_{l} d r^{\prime}$

2. The eigenvalue equation is either

or

$$
\begin{aligned}
& \Phi_{1}\left(\begin{array}{c}
\infty \\
0,0
\end{array}\right)=0, \\
& F_{1}\left(\begin{array}{c}
\infty \\
0,0
\end{array}\right)=0 .
\end{aligned}
$$

Here we can use both expressions for the wave function or the eigenvalue equation, as $F_{1}\left(\begin{array}{c}r \\ 0,0\end{array}\right)$ is well defined, because $K_{l+\frac{1}{2}}(\kappa r)$ has no zeros for $l>-\frac{1}{2}$ and $r>0,[4]$ p. 62 .

3. Sufficient condition for the convergence of $\Phi_{1}\left(\begin{array}{c}r \\ 0,0\end{array}\right)$ or $F_{1}\left(\begin{array}{c}r \\ 0,0\end{array}\right)$. A sufficient condition for the convergence of $F_{1}\left(\begin{array}{c}r \\ 0,0\end{array}\right)$ is that

$$
\bar{q}=\int_{0}^{\infty}\left|U C_{l}^{2}\right| d r \int_{0}^{r} \frac{d r^{\prime}}{C_{l}^{2}}=\int_{0}^{\infty}\left|U S_{l} C_{l}\right| d r<\infty
$$


If we take into account the behaviour of $S_{l}(r) C_{l}(r)$ at $r \rightarrow 0$ and $r \rightarrow \infty$, see f.e. [4] pp. 5, 85 and 86 , we can express (21) as

$$
\begin{aligned}
& \int_{0}^{a} r|V(r)| d r<\infty \\
& \int_{a}^{\infty}|V(r)| d r<\infty
\end{aligned}
$$

and

for any $0<a<\infty$.

On the other hand from (10) we have:

$$
\Phi_{1}\left(\begin{array}{c}
\infty \\
0,0
\end{array}\right)=e^{-\int_{0}^{\infty} U S_{l} C_{l} d r} \cdot F_{1}\left(\begin{array}{c}
\infty \\
0,0
\end{array}\right) .
$$

Therefore condition (21), or (22a) and (22b), is also sufficient for the convergence of $\Phi_{1}\left(\begin{array}{c}\infty \\ 0,0\end{array}\right)$.

One potential satisfying (22a) and (22b) is:

$$
V(r)=\frac{1}{r^{2-s}}, \quad 0<s<1 .
$$

Indeed for this potential we have, [5],

$$
\begin{aligned}
\int_{0}^{\infty}\left|V(r) S_{l}(r) C_{l}(r)\right| d r & =\int_{0}^{\infty} r^{s-1} I_{l+\frac{1}{2}}(\kappa r) K_{l+\frac{1}{2}}(\kappa r) d r \\
& =\frac{\Gamma\left(l+\frac{1+s}{2}\right) B(1-s, s / 2)}{2^{2-s} \kappa^{s} \Gamma\left(l+\frac{3-s}{2}\right)}<\infty .
\end{aligned}
$$

\section{Scattering Problems}

1. In that case the problem is an initial and not a boundary value problem. Thus the only condition we need is that the solution is regular at the origin, i.e.

$$
w_{l}(r) \underset{r \rightarrow 0}{\longrightarrow} r^{l+1}
$$

Therefore it is enough to choose

$$
\begin{aligned}
u_{1}(r)=S_{l}(r) & =\sqrt{\frac{\pi r}{2}} J_{l+\frac{1}{2}}(k r) \\
k^{2} & =\frac{2 m E}{\hbar^{2}}
\end{aligned}
$$


while $u_{2}(r)=C_{l}(r)$ will be chosen properly, for each type of solution, so that

$$
W\left(S_{l}, C_{l}\right)=1 .
$$

The solutions then are obtained from (4) or (7) by putting

$$
\begin{aligned}
u_{1} & =S_{l}, & u_{2} & =C_{l} \\
C & =-U(r), & a & =0 .
\end{aligned}
$$

For the Type I solution we take

$$
C_{l}(r)=\sqrt{\frac{\pi r}{2}} Y^{l+\frac{1}{2}}(k r)
$$

while for the Type II we choose

$$
\bar{C}_{l}(r)=\frac{1}{i} \sqrt{\frac{\pi r}{2}} H_{l+\frac{1}{2}}^{(1)}(k r)
$$

so the central function $F_{1}\left(\begin{array}{c}r \\ 0,0\end{array}\right)$ is well defined, as $H_{l+\frac{1}{2}}^{(1)}(\mathrm{kr})$ has no zeros for $l>-\frac{1}{2}$ and $r>0$, [4], p. 62 .

2. Phase shift: We use the formula:

$$
\tan \eta_{l}=\left.\frac{W\left[w_{l}(r), r^{\frac{1}{2}} J_{l+\frac{1}{2}}(k r)\right]}{W\left[w_{l}(r), r^{\frac{1}{2}} Y_{l+\frac{1}{2}}(k r)\right]}\right|_{r=\infty}
$$

and we obtain

Type I:

$$
\tan \eta_{l}=\frac{e^{-2 f(\infty)} \int_{0}^{\infty} U(r) S_{l}^{2}(r) e^{2 f(r)} \Phi_{1}\left(\begin{array}{c}
r \\
0,0
\end{array}\right) d r}{\Phi_{1}\left(\begin{array}{c}
\infty \\
0,0
\end{array}\right)} .
$$

Type II:

$$
\tan \eta_{l}=-\frac{\int_{0}^{\infty} U(r) S_{l}(r) \bar{C}_{l}(r) d r \int_{0}^{r} \frac{F_{1}\left(\begin{array}{c}
r^{\prime} \\
0,0
\end{array}\right)}{\bar{C}_{l}\left(r^{\prime}\right)^{2}} d r^{\prime}}{F_{1}\left(\begin{array}{c}
\infty \\
0,0
\end{array}\right)-i \int_{0}^{\infty} U(r) S_{l}(r) \bar{C}_{l}(r) d r \int_{0}^{r} \frac{F_{1}\left(\begin{array}{c}
r^{\prime} \\
0,0
\end{array}\right)}{\bar{C}_{l}\left(r^{\prime}\right)^{2}} d r^{\prime}} .
$$

3. Scattering amplitude:

Type I:

$$
a(l, k)=\frac{2}{k} \frac{e^{-f(\infty)} \int_{0}^{\infty} U(r) S_{l}^{2}(r) e^{2 f(r)} \Phi_{1}\left(\begin{array}{c}
r \\
0,0
\end{array}\right) d r}{e^{f(\infty)} \Phi_{1}\left(\begin{array}{c}
\infty \\
0,0
\end{array}\right)-i e^{-f(\infty)} \int_{0}^{\infty} U(r) S_{l}^{2}(r) e^{2 f(r)} \Phi_{1}\left(\begin{array}{c}
r \\
0,0
\end{array}\right) d r}
$$


Type II:

$$
a(l, k)=\frac{2}{k} \frac{\int_{0}^{\infty} U(r) S_{l}(r) \bar{C}_{l}(r) d r \int_{0}^{r} \frac{F_{1}\left(\begin{array}{c}
r^{\prime} \\
0,0
\end{array}\right)}{\bar{C}_{l}\left(r^{\prime}\right)} d r^{\prime}}{F_{1}\left(\begin{array}{c}
\infty \\
0,0
\end{array}\right)} .
$$

4. Sufficient conditions for the convergence of $\Phi_{1}\left(\begin{array}{c}\mathrm{r} \\ 0,0\end{array}\right)$ and $F_{1}\left(\begin{array}{c}r \\ 0,0\end{array}\right)$. (a) If conditions (22a) and (22b), for the potential $U(r)$ are valid, then it is easy to show that:

$$
\bar{q}=\int_{0}^{\infty}|U(r)| C_{l}^{2}(r) e^{-2 f} d r \int_{0}^{r}\left|U\left(r^{\prime}\right)\right| S_{l}^{2}\left(r^{\prime}\right) e^{2 f} d r^{\prime}<\infty
$$

and therefore $\Phi_{1}\left(\begin{array}{c}r \\ 0,0\end{array}\right)$ is convergent for any $0 \leqq r \leqq \infty$.

We first remark that, under conditions (22a) and (22b),

$$
|f(r)|=\left|\int_{0}^{r} U\left(r^{\prime}\right) S_{l}\left(r^{\prime}\right) C_{l}\left(r^{\prime}\right) d r^{\prime}\right|
$$

is bound for $0 \leqq r \leqq \infty$.

Then, if $|f(r)|<\mu$

$$
\begin{aligned}
& \bar{q} \leqq e^{4 \mu} \int_{0}^{\infty}|U(r)| C_{l}^{2}(r) d r \int_{0}^{r}\left|U\left(r^{\prime}\right)\right| S_{l}^{2}\left(r^{\prime}\right) d r^{\prime} \\
& =e^{4 \mu}\left[\int_{0}^{a}|U(r)| C_{l}^{2}(r) d r \int_{0}^{r}|U(r)| S_{l}^{2}\left(r^{\prime}\right) d r^{\prime}\right. \\
& \left.+\int_{a}^{\infty}|U(r)| C_{l}^{2}(r) d r \int_{0}^{r}\left|U\left(r^{\prime}\right)\right| S_{l}^{2}\left(r^{\prime}\right) d r^{\prime}\right] \\
& \leqq e^{4 \mu}\left[\int_{0}^{a}|U(r)| C_{l}^{2}(r) d r \int_{0}^{r}\left|U\left(r^{\prime}\right)\right| S_{l}^{2}\left(r^{\prime}\right) d r^{\prime}\right. \\
& \left.+\int_{a}^{\infty}|U(r)| C_{l}^{2}(r) d r \int_{0}^{\infty}\left|U\left(r^{\prime}\right)\right| S_{l}^{2}\left(r^{\prime}\right) \mid d r^{\prime}\right] .
\end{aligned}
$$

For any $0<a<\infty$, condition (22a) guarantees the convergence of the first term in the bracket, while both conditions are enough for the convergence of the second term.

Therefore $\Phi_{1}\left(\begin{array}{c}\mathrm{r} \\ 0,0\end{array}\right)$ is convergent for any potential $U(r)$ which fulfills conditions (22a) and (22b). 
(b) For the convergence of $F_{1}\left(\begin{array}{c}r \\ 0,0\end{array}\right)$ it is sufficient to demand:

or, from (27)

$$
\bar{p}=\int_{0}^{\infty}\left|U(r) \bar{C}_{l}(r)^{2}\right| d r \int_{0}^{r} \frac{d r^{\prime}}{\left|\bar{C}_{l}\left(r^{\prime}\right)^{2}\right|}<N<\infty
$$

$$
\bar{p}=\int_{0}^{\infty}|U(r)| r\left(J_{v}^{2}+Y_{v}^{2}\right) d r \int_{0}^{r} \frac{d r^{\prime}}{r^{\prime}\left(J_{v}^{2}+Y_{v}^{2}\right)}, \quad v=l+\frac{1}{2} .
$$

For $v=\frac{1}{2}(l=0)$ it is obvious that

$$
\bar{p} \leqq \int_{0}^{\infty} r|U(r)| d r .
$$

For $v>\frac{1}{2}(l>0), r\left[J_{v}^{2}+Y_{v}^{2}\right]$ is a decreasing function, [6]. Therefore

$$
\bar{p} \leqq \int_{0}^{\infty}|U| r\left(J_{v}^{2}+Y_{v}^{2}\right) \frac{r}{r\left[J_{v}^{2}+Y_{v}^{2}\right]}-d r=\int_{0}^{\infty} r|U(r)| d r .
$$

So for the convergence of $F_{1}\left(\begin{array}{c}r \\ 0,0\end{array}\right)$ it is enough to have,

$$
\int_{0}^{\infty} r|U(r)| d r<N<\infty
$$

\section{Concluding Remarks}

In spite of the fact that we accepted a rather strong sufficient condition for the convergence of the series, which form the central functions $\Phi_{1}\left(\begin{array}{c}r \\ 0,0\end{array}\right)$ and $F_{1}\left(\begin{array}{c}r \\ 0,0\end{array}\right)$ we arrived at a loose restriction on the potential. In the case of Type I solutions the restrictions on the potential are:

$$
\int_{0}^{a} r|V(r)| d r<\infty
$$

and

$$
\int_{a}^{\infty}|V(r)| d r<\infty
$$

for any $0<a<\infty$. These conditions, to our knowledge, seem to be weaker than any previous one imposed in similar problems $[2,7]$. In the case of Type II solutions, we derive the restriction on the potential

$$
\int_{0}^{\infty} r|V(r)| d r<\infty
$$

for scattering problems, and the conditions (22) for bound state problems. 
The solution of Type II is preferable in the case of a discrete spectrum, while for the continuum, solution of Type I is preferable.

Numerical applications of the method to various problems is the subject of a forthcoming paper.

Acknowledgments. The authors would like to thank Dr. J. E. Bowcock for some stimulating remarks. One of the authors (Th. K.) would like to express his gratitude to Professor Heisenberg for his hospitality to the Max-Planck-Institut during the period April to November 1969, when this work was initiated. Also he would like to thank the Deutsche Forschungsgemeinschaft for a grant.

\section{References}

1. Kanellopoulos, E.J., Kanellopoulos, Th.V., Wildermuth,K.: Commun. math. Phys. 24, 225 (1972).

2. Newton, R. G.: J. Math. Phys. Vol.1, 319 (1960).

3. See for example Squires, E.: Complex angular momenta and particle physics. Frontiers in Physics. New York-Amsterdam: W. A. Benjamin Inc. 1963.

4. Bateman Project Higher transcendental functions, Vol. II. New York: Mc Graw Hill Book Co. Inc. 1958.

5. Bateman Project Tables of integral transforms, Vol. I. p. 333 (43). New York: Mc Graw Hill Book Co. Inc. 1953.

6. Watson, G. N.: Theory of Bessel functions, Second Ed., p. 446. Cambridge: University Press 1958.

7. Ta-You-Wu, Ohmura, T.: Quantum theory of scattering, p. 45. In this book further references are found. Englewood Cliffs, N. J.: Prentice Hall International 1962.

Th. Kanellopoulos

Institut $\mathrm{f}$. Theoretische Physik

Universität Tübingen

D-7400 Tübingen, Köstlinstr. 6 